\title{
Luz azul emitida pelos dispositivos digitais e suas consequências oftalmológicas: uma revisão integrativa da literatura
}

\author{
Blue light emitted by digital devices and their ophthalmological consequences: an integrative
} literature review

Luz azul emitida por dispositivos digitales y sus consecuencias oftalmológicas: una revisión integradora de la literatura

Recebido: 22/11/2021 | Revisado: 02/12/2021 | Aceito: 11/12/2021 | Publicado: 19/12/2021

\author{
Bruno Sousa Borges \\ ORCID: https://orcid.org/0000-0002-3326-9554 \\ Universidade do Estado de Mato Grosso, Brasil \\ E-mail: bruno.sousa@unemat.br \\ Simone Galli Rocha Bragato \\ ORCID: https://orcid.org/0000-0001-6330-216X \\ Universidade do Estado de Mato Grosso, Brasil \\ E-mail: simone-galli@hotmail.com \\ Heloisa Miura \\ ORCID: https://orcid.org/0000-0001-7198-2281 \\ Universidade do Estado de Mato Grosso, Brasil \\ E-mail: heloisa.miura@unemat.br \\ Larissa Pereira Cabral Corrêa \\ ORCID: https://orcid.org/0000-0001-6930-0667 \\ Centro Oftalmológico Hospital Dia, Brasil \\ E-mail: larissa_correa2010@hotmail.com \\ Victor César Silva Fagundes \\ ORCID: https://orcid.org/0000-0001-8110-188X \\ Universidade do Estado de Mato Grosso, Brasil \\ E-mail: victor.fagundes@unemat.br \\ Ediane Teixeira Lima Silva \\ ORCID: https://orcid.org/0000-0001-6285-5424 \\ Universidade do Estado de Mato Grosso, Brasil \\ E-mail: ediane.teixeira@unemat.br \\ Jordana Gasparelo Santi \\ ORCID: https://orcid.org/0000-0002-7620-4777 \\ Universidade do Estado de Mato Grosso, Brasil \\ E-mail: jordana.santi@unemat.br \\ Guilherme Sampaio Silva \\ ORCID: https://orcid.org/0000-0002-8353-7756 \\ Universidade do Estado de Mato Grosso, Brasil \\ E-mail: guilherme.sampaio@unemat.br
}

\begin{abstract}
Resumo
Objetivo: Caracterizar o impacto da exposição excessiva à luz azul emitida pelos dispositivos digitais na visão dos adolescentes e adultos jovens. Métodos: Revisão integrativa da literatura realizada nas bases de dados BVS, PubMed, SciELO e Periódico Capes. As buscas abrangeram o período de 2016-2021, sendo selecionados os artigos completos e originais envolvendo grupos de adolescentes ou adultos jovens. Resultados: Foram selecionados seis artigos constituídos de estudos transversais descritivos observacionais com questionário autoaplicável, apresentando uma média de 403 participantes na faixa etária entre 18 e 31 anos, sendo a maioria do sexo feminino. Apenas quatro estudos estabeleceram uma relação entre uso prolongado de dispositivos digitais e as manifestações da síndrome da visão computacional, com variações no tempo de uso de telas. Conclusão: Não há um consenso na literatura demonstrando a partir de qual período de exposição seria prejudicial para o surgimento das manifestações oculares, sendo necessário mais estudos considerando os aspectos ambientais e comportamentais de cada indivíduo.
\end{abstract}

Palavras-chave: Luz azul; Síndrome da visão computacional; Manifestações oculares.

\section{Abstract}

Objective: To characterize the impact of excessive exposure to blue light emitted by digital devices on the vision of teenagers and young adults. Methods: Integrative literature review carried out in the BVS, PubMed and Capes Journal databases. The searches covered the period 2016-2021, with complete and original articles involving selected groups of teenagers or young adults. Results: Six articles were selected, consisting of observational descriptive cross-sectional 
studies with a self-administered questionnaire, with an average of 403 members aged between 18 and 31 years old, most of them female. Only four studies established a correlation between prolonged use of digital devices and the manifestations of computer vision syndrome, with variations in screen use time. Conclusion: There is no consensus in the literature showing which period of exposure would be harmful for the appearance of ocular manifestations, requiring further studies considering the environmental and behavioral aspects of each individual.

Keywords: Blue light; Computer vision syndrome; Eye manifestations.

\section{Resumen}

Objetivo: Caracterizar el impacto de la exposición excesiva a la luz azul emitida por dispositivos digitales en la visión de adolescentes y adultos jóvenes. Métodos: revisión integrativa de la literatura realizada en las bases de datos BVS, PubMed y Capes Journal. Las búsquedas cubrieron el período 2016-2021, seleccionándose artículos completos y originales que involucren a grupos de adolescentes o adultos jóvenes. Resultados: Se seleccionaron seis artículos, que consistieron en estudios observacionales descriptivos de corte transversal con un cuestionario autoadministrado, con un promedio de 403 participantes con edades entre 18 y 31 años, la mayoría mujeres. Solo cuatro estudios establecieron una relación entre el uso prolongado de dispositivos digitales y las manifestaciones del síndrome de visión por computadora, con variaciones en el tiempo de uso de la pantalla. Conclusión: No existe consenso en la literatura que demuestre qué período de exposición sería perjudicial para la aparición de manifestaciones oculares, requiriendo más estudios considerando los aspectos ambientales y conductuales de cada individuo.

Palabras clave: Luz azul; Síndrome de visión por computadora; Manifestaciones oculares.

\section{Introdução}

A luz azul é a radiação eletromagnética de comprimento de onda curto (400-500 nanômetros) no espectro visível (400700 nanômetros) que transporta a maior quantidade de energia por fóton, sendo tratada como luz visível de alta energia. Com a popularidade crescente dos terminais de exibição visual que emitem luz azul, como smartphones móveis, tablets ultraportáteis e telas de computador, o seu uso tem chamado cada vez mais a atenção de pesquisadores devido a hipóteses que sugerem um risco potencial de induzir danos fotoquímicos irreversíveis ao tecido ocular (Leung et al., 2017; Niwano et al., 2019; Ouyang et al., 2020; Silva et al., 2015).

Ainda que parte dessa luz azul excessiva seja prejudicial, em níveis adequados é essencial para estabelecer a função visual normal. Além disso, é responsável por desempenhar um papel importante na discriminação de cores e na visão noturna, atua na regulação do relógio biológico circadiano interno humano e mantém um estado de alerta ao estimular as células ganglionares retinais fotossensíveis. Por outro lado, a exposição excessiva do olho à luz azul tende a causar uma série de alterações a nível celular, como estresse oxidativo, apoptose mitocondrial, reação inflamatória e dano ao DNA, resultando no desenvolvimento de erros de refração, olho seco, glaucoma e ceratite (Leung et al., 2017; Ouyang et al., 2020; Zhao et al., 2018).

Dentre as consequências oftalmológicas decorrentes dessas alterações, muitos autores descreveram um conjunto de queixas denominado como Síndrome da Visão Computacional (SVC). É definida como uma síndrome resultante de alterações visuais e oculares relacionadas ao uso de computadores. Outras definições englobam um conjunto de sinais e sintomas oculares e extraoculares associados ao uso prolongado de terminais de exibição visual (Al Tawil et al., 2020; Blehm et al., 2005; Parihar et al., 2016; Ranasinghe et al., 2016; Al Tawil et al., 2020).

A prevalência estimada de SVC mundial é superior a $70 \%$ e cerca de $40 \%$ dos adultos e $80 \%$ dos adolescentes relatam sintomas significativos associados ao uso de dispositivos digitais. As manifestações incluem fadiga ocular, desconforto ocular, olhos cansados, cefaleia, visão turva, visão dupla e olhos secos. Tais achados foram classificados em externos, associados a olhos secos (ardor, lacrimejamento, olhos secos, irritação, cefaleia) ou internos, relacionados a alterações da visão refrativa, acomodativa ou binocular. Além disso, engloba sintomas extraoculares envolvendo sistema musculoesquelético, sistema nervoso periférico e tegumentar. No entanto, os sintomas oculares são as queixas mais frequentemente encontradas entre os usuários (Parihar et al., 2016; Sá, 2016; Sheppard \& Wolffsohn, 2018; Yammouni \& Evans, 2020).

Dada a alta prevalência dessa síndrome e o uso quase universal dos dispositivos digitais, especialmente no campo da mídia móvel, o presente estudo tem como objetivo analisar o impacto da exposição de luz azul emitida pelos dispositivos 
eletrônicos e as consequências oftalmológicas na visão de adolescentes e adultos jovens.

\section{Metodologia}

Com o objetivo de obter os resultados e respostas acerca da problematização apresentada neste trabalho, foi realizada uma revisão de literatura integrativa tendo como propósito a síntese e discussão crítica dos resultados obtidos em pesquisas relacionadas ao tema. Tal método permite uma abordagem metodológica referente às revisões, permitindo a inclusão de estudos experimentais e não-experimentais para uma compreensão completa do fenômeno analisado (Souza et al., 2010).

Tipo de Estudo: Revisão integrativa sobre o impacto da luz azul emitida pelos dispositivos digitais e suas consequências oftalmológicas.

Fontes de Dados: A coleta de dados foi realizada nas bases de dados BVS, PubMed, SciELO e Periódico Capes.

Coleta de Dados: Foram consultados estudos dos últimos seis anos, entre o período de 2016-2021, a fim de avaliar as possíveis consequências oftalmológicas decorrentes do uso excessivo e prolongado de telas emissoras de luz azul.

Critérios de Inclusão: Artigos completos e originais; Período de 2016-2021; Grupos de adolescentes e adultos jovens.

Critérios de Exclusão: Crianças; Trabalhos anteriores à 2016; Artigos duplicados entre as bases de dados.

Descritores: Manifestações oculares; Adolescentes; Adultos jovens e seus correspondentes em inglês e espanhol: Ocular manifestations; Teenagers; Young adults; Manifestaciones oculares; Adolescentes; Adultos jovenes.

Termos Livres: Luz azul; Dispositivos digitais; Síndrome da visão computacional; Tensão ocular digital e seus correspondentes em inglês e espanhol: Blue light; Digital devices; Computer vision syndrome; Digital eye strain; Luz azul; Dispositivos digitales; Síndrome de visión por computadora; Fatiga visual digital.

\section{Resultados}

Foram classificados um total de noventa e nove artigos com evidências de relação entre exposição à luz azul emitida pelos dispositivos eletrônicos e suas possíveis consequências oftalmológicas. Ao final, apenas seis estudos foram incluídos por se enquadrarem dentro dos critérios de inclusão, conforme mostra a Figura 1, elaborada de acordo com o fluxograma PRISMA (Galvão et al., 2015). 
Figura 1 - Fluxograma de identificação e seleção dos artigos nas bases de dados para a revisão integrativa.

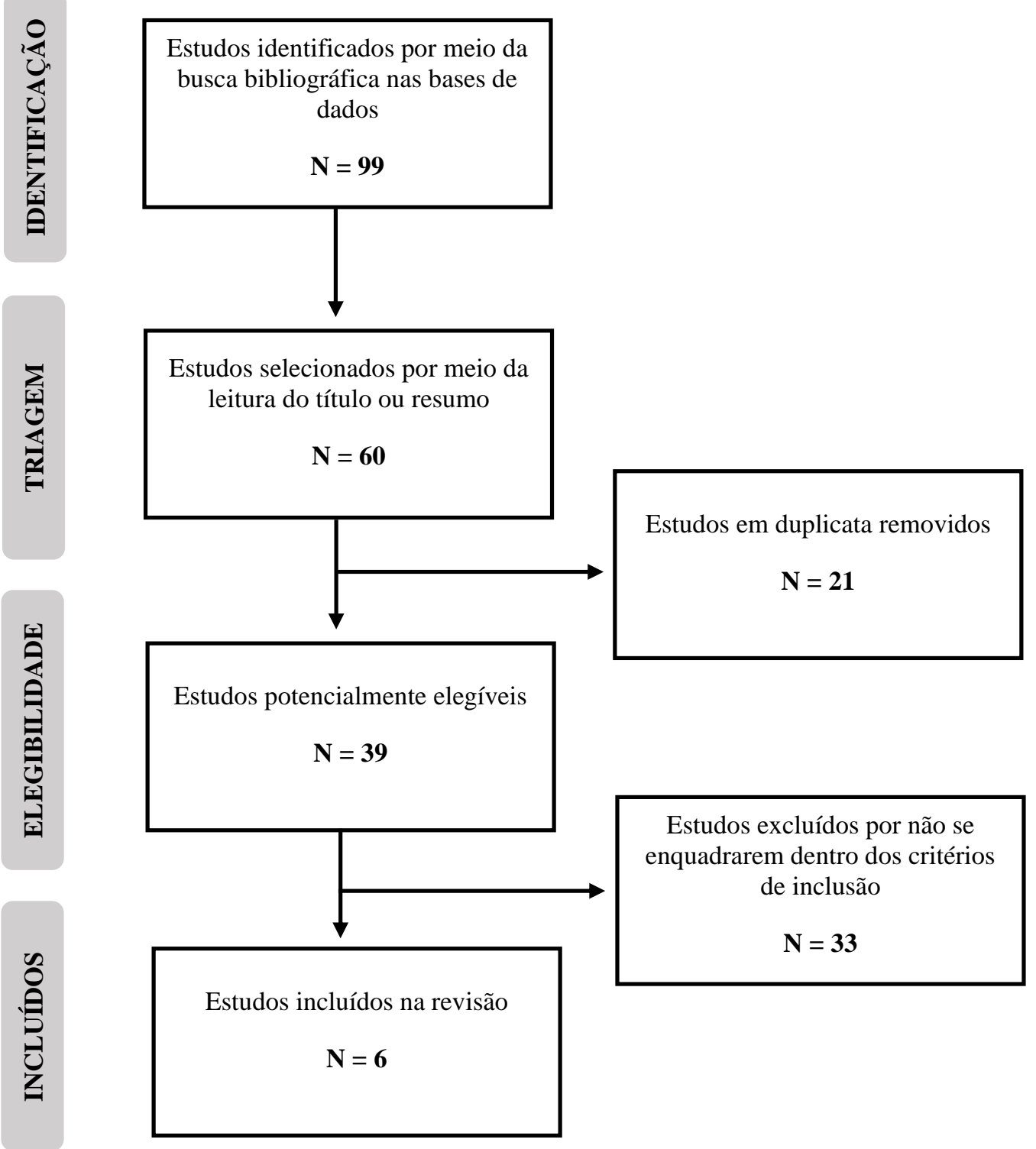

Fonte: Adaptado do fluxograma PRISMA. Elaborado pelos autores (2021).

A seguir, no Quadro 1, são apresentados os seis artigos incluídos nesta revisão integrativa, sendo interpretados e sintetizados através da análise dos resultados. 
Quadro 1 - Características gerais dos artigos selecionados para amostra da revisão integrativa, de acordo com autores, ano de publicação, tipo e local de estudo, população estudada, faixa etária, tempo aproximado do uso de telas e sintomas associados.

\begin{tabular}{|c|c|c|c|c|c|c|}
\hline $\begin{array}{c}\text { AUTORES, ANO } \\
\text { DE } \\
\text { PUBLICAÇÃO }\end{array}$ & $\begin{array}{l}\text { TIPO DE } \\
\text { ESTUDO }\end{array}$ & $\begin{array}{l}\text { LOCAL } \\
\text { DE } \\
\text { ESTUDO }\end{array}$ & $\begin{array}{l}\text { POPULAÇÃO } \\
\text { ESTUDADA }\end{array}$ & $\begin{array}{c}\text { FAIXA } \\
\text { ETÁRIA }\end{array}$ & $\begin{array}{c}\text { TEMPO } \\
\text { APROXIMADO } \\
\text { DO USO DE } \\
\text { TELAS }\end{array}$ & $\begin{array}{c}\text { SINTOMAS } \\
\text { ASSOCIADOS }\end{array}$ \\
\hline Gammoh, 2021 & $\begin{array}{c}\text { Estudo } \\
\text { transversal }\end{array}$ & Jordânia & $\begin{array}{l}\mathrm{N}=382 \text { alunos de } \\
\text { graduação; } 60,9 \% \\
\text { mulheres e } 39,1 \% \\
\text { homens }\end{array}$ & $\begin{array}{c}\text { Idade } \\
\text { média: } \\
21,5 \text { anos } \\
\text { (entre 18- } \\
24 \text { anos) }\end{array}$ & $\begin{array}{c}55,5 \% \text { utilizaram } \\
\text { dispositivos } \\
\text { digitais }>6 \\
\text { horas/dia }\end{array}$ & $\begin{array}{c}\text { Lacrimejamento } \\
(59 \%) \text {; cefaleia } \\
(53 \%) \text {; fotofobia } \\
(51 \%) ; \text { pálpebras } \\
\text { pesadas }(29 \%) \text {; visão } \\
\text { dupla }(18,3 \%)\end{array}$ \\
\hline Altalhi et al., 2020 & $\begin{array}{c}\text { Estudo } \\
\text { transversal }\end{array}$ & $\begin{array}{l}\text { Arábia } \\
\text { Saudita }\end{array}$ & $\begin{array}{c}\mathrm{N}=334 \text { alunos de } \\
\text { graduação; } 55,4 \% \\
\text { homens e } 44,6 \% \\
\text { mulheres }\end{array}$ & $\begin{array}{c}\text { Idade } \\
\text { média: } 20 \\
\text { anos }\end{array}$ & $\begin{array}{c}\text { Maioria dos } \\
\text { estudantes } \\
\text { utilizaram } \\
\text { dispositivos } \\
\text { digitais > } 6 \\
\text { horas/dia (não foi } \\
\text { relatada a } \\
\text { porcentagem exata) }\end{array}$ & $\begin{array}{c}\text { Cefaleia }(68 \%) \text {; perda } \\
\text { transitória da visão } \\
(65 \%) \text {; coceira nos } \\
\text { olhos }(63 \%) ; \\
\text { pálpebras pesadas } \\
(9 \%)\end{array}$ \\
\hline $\begin{array}{c}\text { Al Rashidi e } \\
\text { Alhumaidan, } 2017\end{array}$ & $\begin{array}{c}\text { Estudo } \\
\text { transversal }\end{array}$ & $\begin{array}{l}\text { Arábia } \\
\text { Saudita }\end{array}$ & $\begin{array}{l}\mathrm{N}=634 \text { alunos de } \\
\text { graduação; } \\
77,28 \% \text { homens e } \\
22,71 \% \text { mulheres }\end{array}$ & $\begin{array}{c}\text { Idade } \\
\text { média: } \\
21,40 \\
\text { (entre 18- } \\
25 \text { anos) }\end{array}$ & $\begin{array}{c}58,51 \% \text { utilizaram } \\
\text { dispositivos } \\
\text { digitais }>8 \\
\text { horas/dia }\end{array}$ & $\begin{array}{c}\text { Fadiga ocular } \\
(62,14 \%) \text { e ardor nos } \\
\text { olhos }(7,57 \%) \text {. Outros } \\
\text { sintomas menos } \\
\text { comuns: vermelhidão, } \\
\text { olhos secos e } \\
\text { lacrimejamento (não } \\
\text { foram relatadas as } \\
\text { frequências) }\end{array}$ \\
\hline Mowat et al., 2018 & $\begin{array}{c}\text { Estudo } \\
\text { transversal }\end{array}$ & Jamaica & $\begin{array}{l}\mathrm{N}=409 \text { alunos de } \\
\text { graduação; } 78 \% \\
\text { mulheres e } 22 \% \\
\text { homens }\end{array}$ & $\begin{array}{c}\text { Idade } \\
\text { média: } \\
21,6 \text { (entre } \\
\text { 18-33 } \\
\text { anos) }\end{array}$ & $\begin{array}{c}\text { 40,3\% utilizaram } \\
\text { dispositivos } \\
\text { digitais }>6 \\
\text { horas/dia; 33,3\% } \\
\text { entre 4-6 horas/dia; } \\
5,6 \%<2 \text { horas/dia }\end{array}$ & $\begin{array}{c}\text { Dor no pescoço } \\
(75,1 \%) \text {, fadiga ocular } \\
(67 \%) \text {, dor no ombro } \\
(65,5 \%) \text {, ardor nos } \\
\text { olhos }(61,9 \%) \text {, visão } \\
\text { turva }(51,6 \%) \text {, visão } \\
\text { dupla }(28,9 \%), \text { olhos } \\
\text { secos }(26,2 \%)\end{array}$ \\
\hline
\end{tabular}




\begin{tabular}{|c|c|c|c|c|c|c|}
\hline $\begin{array}{c}\text { Moldovan et al., } \\
2019\end{array}$ & $\begin{array}{c}\text { Estudo } \\
\text { transversal }\end{array}$ & Romênia & $\begin{array}{l}\mathrm{N}=420 \text { alunos de } \\
\text { graduação; } 60 \% \\
\text { mulheres e } 40 \% \\
\text { homens }\end{array}$ & $\begin{array}{l}\text { Entre } 21- \\
23 \text { anos }\end{array}$ & $\begin{array}{c}65 \% \text { utilizaram } \\
\text { dispositivos digitais } \\
<5 \text { horas/dia }\end{array}$ & $\begin{array}{c}\text { Fadiga ocular } \\
(86,1 \%) ; \text { ardor nos } \\
\text { olhos }(46,8 \%) ; \\
\text { cefaleia }(46,8 \%) ; \\
\text { vermelhidão (43\%); } \\
\text { fotofobia (41,8\%); } \\
\text { espasmo palpebral } \\
\text { (32,9\%); olhos secos, } \\
\text { lacrimejamento ou } \\
\text { coceira nos olhos } \\
(32,9 \%)\end{array}$ \\
\hline $\begin{array}{c}\text { Cantó-Sancho et } \\
\text { al., } 2021\end{array}$ & $\begin{array}{c}\text { Estudo } \\
\text { transversal }\end{array}$ & Espanha & $\begin{array}{l}\mathrm{N}=244 \text { alunos de } \\
\text { graduação; } 57 \% \\
\text { mulheres e } 43 \% \\
\text { homens }\end{array}$ & $\begin{array}{c}\text { Idade } \\
\text { média: } \\
20,7 \text { anos } \\
\text { (entre 18- } \\
29 \text { anos) }\end{array}$ & $\begin{array}{l}\text { 61,5\% utilizaram } \\
\text { dispositivos digitais } \\
\text { entre } 4-8 \text { horas/dia }\end{array}$ & $\begin{array}{c}\text { Cefaleia }(78,7 \%) ; \\
\text { coceira nos olhos } \\
(73 \%) ; \text { pálpebras } \\
\text { pesadas }(61,9 \%) ; \\
\text { perda transitória da } \\
\text { visão }(60,3 \%) ; \text { halos } \\
\text { coloridos em torno de } \\
\text { objetos }(21,3 \%) \text { e } \\
\text { visão dupla }(8,6 \%)\end{array}$ \\
\hline
\end{tabular}

Fonte: Elaborado pelos autores (2021).

Em síntese, as pesquisas selecionadas constituíram-se de estudos transversais descritivos observacionais realizados em diferentes localidades, através de questionário autoaplicável contendo informações demográficas (idade e sexo), duração de uso dos dispositivos digitais e frequência de sintomas oculares. As literaturas avaliadas apresentaram uma média de 403 participantes na faixa etária entre 18 e 31 anos, sendo a maioria correspondente ao sexo feminino.

Além disso, os estudos avaliados relataram diferentes dados quanto ao tempo de exposição aos dispositivos digitais. $\mathrm{O}$ estudo de Gammoh (2021) indica que 55,5\% dos universitários utilizaram os dispositivos digitais mais de 6 horas por dia, assim como o estudo de Mowatt et al (2018) e Altalhi et al (2020). O primeiro observou que 40,3\% dos entrevistados utilizaram telas por esse mesmo período, enquanto o último não definiu a porcentagem exata de uso entre os universitários.

O estudo de Gammoh (2021) apresentou como principais manifestações oculares o lacrimejamento (59\%), cefaleia $(53 \%)$ e fotofobia $(51 \%)$, além de pálpebras pesadas (29\%) e visão dupla (18,3\%) como sintomas menos frequentes. Por sua vez, o estudo de Altalhi et al (2020) apresentou cefaleia (68\%), perda transitória da visão (65\%) e coceira nos olhos (63\%) como os sintomas mais frequentes, sendo pálpebras pesadas (9\%) a queixa menos relatada. Já o estudo de Mowatt et al (2018) apresentou como sintomas oculares principais a dor no pescoço $(75,1 \%)$, fadiga ocular $(67 \%)$, dor nos ombros $(65,5 \%)$, ardor nos olhos $(61,9 \%)$, visão turva $(51,6 \%)$, enquanto visão dupla $(28,9 \%)$ e olhos secos $(26,2 \%)$ foram as manifestações menos presentes entre os usuários.

Por outro lado, o estudo de Al Rashidi e Alhumaidan (2017) relatou que 58,51\% dos participantes utilizaram os dispositivos por mais de 8 horas por dia, sendo o maior tempo de exposição considerado dentre as literaturas descritas. Nesse estudo as queixas de fadiga ocular $(62,14 \%)$ e ardor nos olhos $(7,57 \%)$ foram as mais descritas pelos usuários, enquanto vermelhidão, olhos secos e lacrimejamento foram menos comuns, porém, não foram relatadas as frequências desses sintomas.

Por fim, os estudos em que os participantes utilizaram os dispositivos digitais por menos tempo foram o de Moldovan 
et al (2019) em que 65\% utilizaram menos de 5 horas por dia e Cantó-Sancho et al (2021) em que 61,5\% utilizaram por um período entre 4 e 8 horas, sendo que desse total apenas 15,6\% utilizaram por mais de 8 horas diária. No primeiro, as principais queixas relatadas foram fadiga ocular $(86,1 \%)$, ardor nos olhos (46,8\%), cefaleia (46,8\%), vermelhidão (43\%), fotofobia (41,8\%) e espasmo palpebral (32,9\%), enquanto olhos secos, lacrimejamento ou coceira ocular foram menos comuns. Já no estudo de Cantó-Sancho et al (2021) foi possível observar cefaleia (78,7\%), coceira nos olhos (73\%), pálpebras pesadas (61,9\%) e perda transitória da visão $(60,3 \%)$ com mais frequência e halos coloridos em torno de objetos $(21,3 \%)$ e visão dupla $(8,6 \%)$ como queixas clínicas menos frequentes.

\section{Discussão}

Do total de artigos incluídos, apenas quatro dos seis estabeleceram uma relação entre o uso prolongado de dispositivos digitais e as manifestações oculares da SVC, tendo limitações na avaliação desses dados, uma vez que as informações foram autorrelatadas pelos usuários.

No estudo conduzido por Gammoh (2021), a maioria dos alunos (55,5\%) utilizaram os dispositivos digitais por mais de 6 horas por dia, sendo considerado um fator de risco para o desenvolvimento da SVC. Nesse sentido, esse estudo mostra que há uma relação entre horas utilizadas em telas e sintomas da SVC, com 93,9\% dos entrevistados que usaram os dispositivos por esse período sendo portadores da síndrome. Apesar disso, mais da metade $(63,9 \%)$ relataram interrupções no uso dos dispositivos através de pausas pela regra 20-20-20 que consiste em fazer uma pausa após 20 minutos de uso dos dispositivos digitais e olhar a uma distância de 6 metros por um período de 20 segundos, técnica considerada como simples e eficaz para aliviar a fadiga ocular digital e os sintomas da SVC.

Em contrapartida, os estudos de Altalhi et al (2020) e Mowatt et al (2018), realizados com universitários da Arábia Saudita e Romênia, respectivamente, mostraram que a maior duração de uso de dispositivos digitais pelos entrevistados, ou seja, mais de 6 horas por dia, não está significativamente associada a um aumento nos sintomas de SVC. Além disso, esses estudos mostraram que as práticas ergonômicas como as pausas a cada 20 minutos de uso de telas embora fossem utilizadas, não eram bem praticadas e aplicadas pela maioria dos alunos, não sendo, por isso, associadas à diminuição do número de sintomas oculares.

Na abordagem de Al Rashidi e Alhumaidan (2017), 77,76\% da população total do estudo foram considerados míopes, em comparação com o estudo de Gammoh (2021) e Altalhi et al (2020) em que a maioria dos participantes eram emétropes, o que contribuiu para comprovar que há uma relação estatisticamente significativa entre a miopia e gravidade dos sintomas da SVC. Al Rashidi e Alhumaidan (2017) revelaram ainda que a apresentação da SVC era mais grave naqueles universitários míopes que utilizavam lentes de contato quando comparado com aqueles que usavam apenas óculos. Já no estudo de Altalhi et al (2020) e Cantó-Sancho et al (2021) a prevalência da SVC era maior apenas naqueles universitários que usavam óculos.

Nesse estudo de Al Rashidi e Alhumaidan (2017), a média de uso de dispositivos digitais foi mais de 8 horas por dia, sendo que a maioria dos indivíduos queixaram de fadiga ocular $(62,14 \%)$, corroborando com essa estatística os estudos de Moldovan et al (2019) e Mowatt et al (2018), com 86,1\% e 67\%, respectivamente, sendo a manifestação ocular mais comum entre as literaturas abordadas.

Por fim, é válido ressaltar que as diferenças entre os dois gêneros permaneceram constantes entre os estudos avaliados, uma vez que as mulheres apresentaram o maior número de sintomas da SVC. No estudo realizado por Mowatt et al (2018) as mulheres apresentaram uma razão de chances de 2,6 vezes mais predispostas a desenvolver a SVC. Já no estudo de Altalhi et al (2020) foi relatado que os homens apresentaram uma prevalência maior em alguns sintomas particulares, como olhos secos, vermelhidão nos olhos e visão turva. 


\section{Considerações Finais}

Apesar do uso de telas por tempo prolongado ser considerado um fator de risco para o desenvolvimento da síndrome, não há um consenso na literatura demonstrando a partir de qual período de exposição seria prejudicial para o surgimento das manifestações oculares. É válido ressaltar que os participantes foram solicitados a apresentar uma média do número de horas utilizadas em telas, uma vez que variações individuais são esperadas.

As limitações do presente estudo estão relacionadas pelo fato de que pesquisas subjetivas através de questionários, sem exame oftalmológico minucioso, não são ideais para documentar a prevalência da SVC. Assim, incentiva-se o desenvolvimento de mais estudos e uma análise quantitativa e qualitativa sobre a temática visando fundamentar a relação entre terminais de exibição visual e as consequências oftalmológicas.

\section{Referências}

Al Rashidi, S. H., \& Alhumaidan, H. (2017). Computer vision syndrome prevalence, knowledge and associated factors among Saudi Arabia University Students: Is it a serious problem? International Journal of Health Sciences, 11(5), 17-19.

Al Tawil, L., Aldokhayel, S., Zeitouni, L., Qadoumi, T., Hussein, S., \& Ahamed, S. S. (2020). Prevalence of self-reported computer vision syndrome symptoms and its associated factors among university students. European Journal of Ophthalmology, 30(1), 189-195.

Altalhi, A., Khayyat, W., Khojah, O., Alsalmi, M., \& Almarzouki, H. (2020). Computer Vision Syndrome Among Health Sciences Students in Saudi Arabia: Prevalence and Risk Factors. Cureus, 12(2).

Blehm, C., Vishnu, S., Khattak, A., Mitra, S., \& Yee, R. W. (2005). Computer Vision Syndrome: A Review. Survey of Ophthalmology, 50(3), $253-262$.

Cantó-Sancho, N., Sánchez-Brau, M., Ivorra-Soler, B., \& Seguí-Crespo, M. (2021). Computer vision syndrome prevalence according to individual and video display terminal exposure characteristics in Spanish university students. International Journal of Clinical Practice, 75(3).

Galvão, T. F, Pansani T. de S. A., Harrad D. (2015). Principais itens para relatar Revisões sistemáticas e Meta-análises: A recomendação PRISMA. Epidemiol. Serv. Saúde 24 (2).

Gammoh, Y. (2021). Digital Eye Strain and Its Risk Factors Among a University Student Population in Jordan: A Cross-Sectional Study. Cureus, 13(2), e13575.

Leung, T. W., Li, R. W., \& Kee, C. (2017). Blue-Light Filtering Spectacle Lenses: Optical and Clinical Performances. PLoS ONE, 12(1).

Moldovan, H.-R., Voidazan, S.-T., Moldovan, G., Vlasiu, M.-A., Moldovan, G., \& Panaitescu, R. (2019). Accommodative asthenopia among Romanian computer-using medical students-A neglected occupational disease. Archives of Environmental \& Occupational Health, 75(4), $235-241$.

Mowatt, L., Gordon, C., Santosh, A. B. R., \& Jones, T. (2018). Computer vision syndrome and ergonomic practices among undergraduate university students. International Journal of Clinical Practice, 72(1), e13035.

Niwano, Y., Iwasawa, A., Tsubota, K., Ayaki, M., \& Negishi, K. (2019). Protective effects of blue light-blocking shades on phototoxicity in human ocular surface cells. BMJ Open Ophthalmology, 4(1).

Ouyang, X., Yang, J., Hong, Z., Wu, Y., Xie, Y., \& Wang, G. (2020). Mechanisms of blue light-induced eye hazard and protective measures: A review. Biomedicine \& Pharmacotherapy, 130, 110577.

Parihar, J. K. S., Jain, V. K., Chaturvedi, P., Kaushik, J., Jain, G., \& Parihar, A. K. S. (2016). Computer and visual display terminals (VDT) vision syndrome (CVDTS). Medical Journal, Armed Forces India, 72(3), 270-276.

Ranasinghe, P., Wathurapatha, W. S., Perera, Y. S., Lamabadusuriya, D. A., Kulatunga, S., Jayawardana, N., \& Katulanda, P. (2016). Computer vision syndrome among computer office workers in a developing country: An evaluation of prevalence and risk factors. BMC Research Notes, 9.

Sá, E. C. (2016). Síndrome da visão do computador e função visual em trabalhadores usuários de computador de um hospital público universitário de São Paulo: Prevalência e fatores associados. Doutorado em Saúde Pública, Universidade de São Paulo.

Sheppard, A. L., \& Wolffsohn, J. S. (2018). Digital eye strain: Prevalence, measurement and amelioration. BMJ Open Ophthalmology, 3(1).

Silva, L. C., Maia, L. D., Pinheiro, D. R., Matias, L. da S. M., Salvo, V. F., André, J. de O., \& Foureaux, G. (2015). Correlação entre a exposição diária à luz azul violeta emitida por dispositivos digitais e a visão de adultos jovens. Saúde em Revista, 15(41), 47-55.

Souza, M. T. de, Silva, M. D. da, \& Carvalho, R. de. (2010). Integrative review: What is it? How to do it? Einstein (São Paulo), 8(1), $102-106$.

Yammouni, R., \& Evans, B. J. (2020). An investigation of low power convex lenses (adds) for eyestrain in the digital age (CLEDA). Journal of Optometry, 13(3), 198-209.

Zhao, Z.-C., Zhou, Y., Tan, G., \& Li, J. (2018). Research progress about the effect and prevention of blue light on eyes. International Journal of Ophthalmology, 11(12), 1999-2003. 